\title{
Investigations on Synthesis, Structural, Morphological and Dielectric Properties of Manganese Oxides Nanoparticles
}

\section{Sagadevan $\mathbf{S}^{*}$}

Department of Physics, Sree Sastha Institute of Engineering and Technology, Chembarambakkam, Chennai-600 123, India

\begin{abstract}
Manganese oxides $\left(\mathrm{MnO}_{2}\right)$ nanoparticles were synthesized using a co-precipitation technique. The as prepared nanopowder was used for further characterization. The size, structure and morphology of $\mathrm{MnO}_{2}$ nanoparticles were characterized by X-ray diffraction (XRD) and SEM. The crystallite size of the synthesized $\mathrm{MnO}_{2}$ nanoparticles was obtained from X-ray diffraction study using Debye-Scherer formula and it was found to be $22 \mathrm{~nm}$. The surface morphology of prepared $\mathrm{MnO}_{2}$ nanoparticles was analyzed by scanning electron microscope. The optical properties were analyzed using UV-studies. Dielectric studies were carried out for the pelletized sample of $\mathrm{MnO}_{2}$ nanoparticles. The dielectric properties of $\mathrm{MnO}_{2}$ nanoparticles were studied in the different frequency range of $50 \mathrm{~Hz}-5 \mathrm{MHz}$ at different temperatures. The frequency dependence of the dielectric constant and dielectric loss is found to decrease with an increase in the frequency at different temperatures.
\end{abstract}

Keywords: $\mathrm{MnO}_{2}$ nanoparticles; XRD; SEM; Dielectric constant and dielectric loss

\section{Introduction}

Nano-sized materials are new substances quickly developed and generally measured as 1 to $100 \mathrm{~nm}$ size in one dimension. At a nanometer scale, the surface area increases hastily as particle size decreases, as a result, the electromagnetic, thermal, optical, and mechanical properties of nanomaterials modify. In recent years, manganese oxides $\left(\mathrm{MnO}_{2}\right)$ have concerned considerable research interest due to their characteristic physical and chemical properties and broad applications such as ion exchange, molecular adsorption, energy storage, catalysis, biosensor [1-3]. Manganese oxides have established application in catalysis, ion exchange reactions, as cathode materials for rechargeable batteries and as distinguish agents for magnetic resonance imaging (MRI). The manganese oxide nanoparticles were used to improve the mechanical and thermal properties of polymers and to enhance the ionic conductivity of polymer electrolytes [4-6]. Manganese oxides take place in a variety of oxidation states and chemical and structural forms. Due to their distinctive properties and wide applications, the synthesis of manganese oxide nanoparticles has been investigated in order to put their chemical composition, structure, size and morphology under control $[7,8]$. Several novel and effective routes have been devoted to prepare manganese oxides nanomaterials with various shapes and excellent properties, such as hydrothermal method [9-12], sol-gel synthesis [13], wet chemical route [14,15], pulsed laser deposition method [16], and precursor technique [17]. Co-precipitation method offers advantages like, simple and rapid preparative method, easy control of particle size and composition can be made in this method and also, there are various possibilities to modify the particle surface state and overall homogeneity. In the present study, $\mathrm{MnO}_{2}$ nanoparticles were synthesized by co-precipitation method. Present work reports synthesis of $\mathrm{MnO}_{2}$ nanoparticles and its characterization by XRD, SEM, UV-analysis and Dielectric studies.

\section{Materials and Method}

$\mathrm{MnO}_{2}$ nanoparticles were prepared co-precipitation method by using the manganese (II) sulphate and manganese oxalat in the method reported in literature [18]. The salts were dissolved in de-ionized water and stirred well by magnetic stirrer for $30 \mathrm{~min}$ at a constant temperature $50^{\circ} \mathrm{C}$. Sodium hydroxide solution was added drop wise into the solution. The solution stirring was continued then heated at $60^{\circ} \mathrm{C}$ for $1 \mathrm{hr}$ in a closed vessel. Ethanol was added to the brown coloured solution obtained, and the total volume of the solution was adjusted to $100 \mathrm{ml}$. Precipitates were dried for overnight at $100^{\circ} \mathrm{C}$. Than the precipitates was kept in muffle furnace at $500^{\circ} \mathrm{C}$ for $3 \mathrm{hrs}$. The XRD pattern of the $\mathrm{MnO}_{2}$ nanoparticles was recorded by using a powder X-ray diffractometer (Schimadzu model: XRD 6000 using CuKa $(\lambda=0.154$ $\mathrm{nm}$ ) radiation, with a diffraction angle between $0^{\circ}$ to $80^{\circ}$. The crystallite size was determined from the broadenings of corresponding X-ray spectral peaks by using Debye Scherrer's formula. Scanning Electron Microscopy (SEM) studies were carried out on JEOL, JSM-67001. The optical absorption spectrum of the $\mathrm{MnO}_{2}$ nanoparticles has been taken by using the VARIAN CARY MODEL 5000 spectrophotometer in the wavelength range of 200-600 $\mathrm{nm}$. The dielectric properties of the $\mathrm{MnO}_{2}$ nanoparticles were analyzed using a HIOKI 3532-50 LCR HITESTER over the frequency range $50 \mathrm{~Hz}-5 \mathrm{MHz}$.

\section{Results and Discussion}

\section{$\mathrm{X}$-ray diffraction analysis}

In an X-ray tube, the high voltage maintained across the electrodes draws electrons toward a metal target (the anode). X-rays are produced at the point of impact, and radiate in all directions. X-ray diffraction pattern of the as prepared $\mathrm{MnO}_{2}$ nanoparticles is shown in Figure 1. From the XRD pattern, it is clear from the broadening of diffraction peaks of that the particles crystallize at nanoscale range. The average particle size was calculated using Debye-Scherrer formula,

$$
D=\frac{0.9 \lambda}{\beta \cos \theta}
$$

*Corresponding author: Sagadevan S, Department of Physics, Sree Sastha Institute of Engineering and Technology, Chembarambakkam, Chennai 600123 India, Tel: 0442681 0114; E-mail: sureshsagadevan@gmail.com

Received May 08, 2015; Accepted May 23, 2015; Published May 31, 2015

Citation: Sagadevan S (2015) Investigations on Synthesis, Structural, Morphological and Dielectric Properties of Manganese Oxides Nanoparticles. J Material Sci Eng 4: 172. doi:10.4172/2169-0022.1000172

Copyright: () 2015 Sagadevan S. This is an open-access article distributed under the terms of the Creative Commons Attribution License, which permits unrestricted use, distribution, and reproduction in any medium, provided the original author and source are credited. 
Citation: Sagadevan S (2015) Investigations on Synthesis, Structural, Morphological and Dielectric Properties of Manganese Oxides Nanoparticles. J Material Sci Eng 4: 172. doi:10.4172/2169-0022.1000172

Page 2 of 3

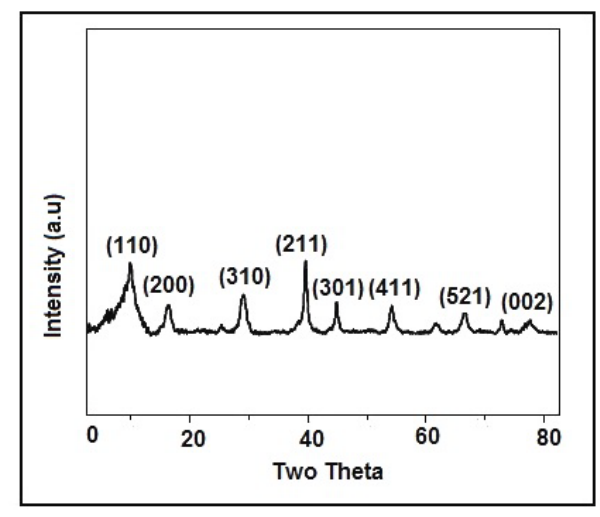

Figure 1: Powder XRD patterns of $\mathrm{MnO}_{2}$ nanoparticles

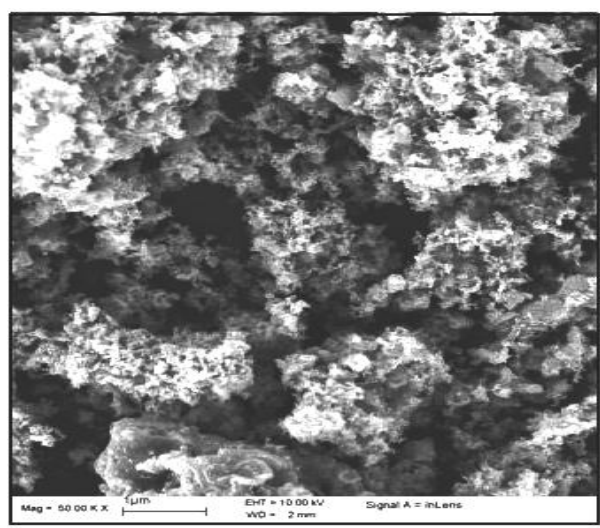

Figure 2: The SEM image of $\mathrm{MnO}_{2}$ nanoparticles.

By Knowing the wavelength $(\lambda)$ full width at half maximum (FWHM) of the peaks $\beta$ and the diffracting angle $\theta$, Particle size (D) was calculated. The calculated average particle size of $\mathrm{MnO}_{2}$ nanoparticles was found to be around $22 \mathrm{~nm}$.

\section{SEM analysis}

Scanning electron microscopy (SEM) was employed to analyze the morphology and the growth features of the aggregates of the as prepared $\mathrm{MnO}_{2}$ nanoparticles. SEM images of as synthesized $\mathrm{MnO}_{2}$ nanoparticles are shown in Figure 2. From the image, it is clear that the particles were highly agglomerated in nature. Due to aggregating or overlapping of smaller particles there are some larger particles. The SEM pictures clearly show randomly distributed grains with smaller size.

\section{UV-visible absorption spectrum}

Ultraviolet spectrometer consists of a light source, reference and samples beams, a monochromator and a detector. The ultraviolet spectrum for a compound is obtained by exposing a sample of the compound to ultraviolet light from a light source. Optical absorption measurement was carried out on nanoparticles. Figure 3 shows the variation of the optical absorbance with the wavelength of the nanoparticles. The optical absorption coefficient has been calculated in the wavelength range of $200-600 \mathrm{~nm}$. The absorption edge has been obtained at a shorter wavelength. The absorption edges are seen to be shifted slightly towards lower wave number (blue shift). This shift indicates an increase in the band gap, which can be attributed to a decrease in particle size. The value of the absorption edge of nanoparticles is $340 \mathrm{~nm}$, blue shift was observed due to the quantum confinement effects.

\section{Dielectric studies}

The dielectric constant was measured as a function of the frequency at different temperatures as shown in Figure 4, while the corresponding dielectric losses are depicted in Figure 5. Figure 4 shows the plot of the dielectric constant versus applied frequency. It is observed that the dielectric constant decreases with increasing frequency and then attains almost a constant value in the high frequency region. This also shows that the value of the dielectric constant increases with an increase in the temperatures. The involvement of the decrease in the dielectric constant due to electronic polarization is quite less. Dipolar polarization is also predictable to decrease with temperature as it is inversely proportional to temperature. The contribution to polarizability of the space charge depends on the purity of the nanoparticles. At low temperature and high frequency, we may take it as small. However, it is significant in the low frequency region. As the temperature increases, the contribution of the space charge effect towards polarization may have a tendency to increase [19].

The dielectric loss considered as a function of frequency at different temperatures is shown in Figure 5. These curves suggest that the

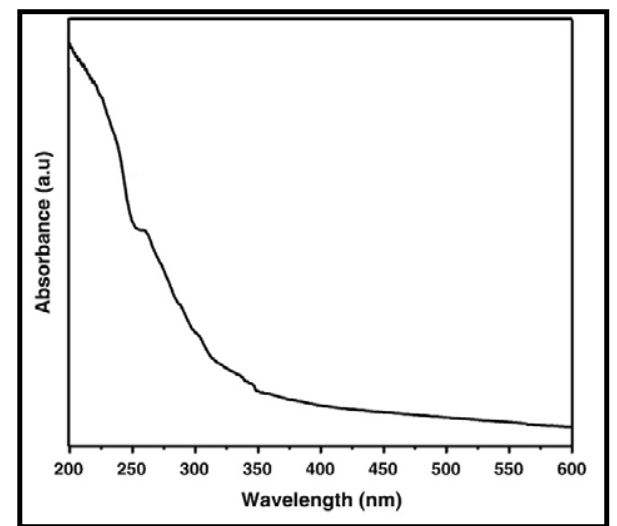

Figure 3: Optical absorption spectrum of $\mathrm{MnO}_{2}$ nanoparticles.

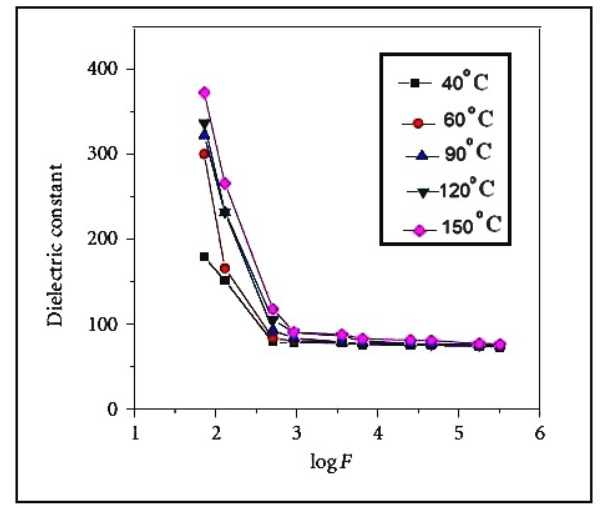

Figure 4: Variation of dielectric constant with frequency. 
Citation: Sagadevan S (2015) Investigations on Synthesis, Structural, Morphological and Dielectric Properties of Manganese Oxides Nanoparticles. J Material Sci Eng 4: 172. doi:10.4172/2169-0022.1000172

Page 3 of 3

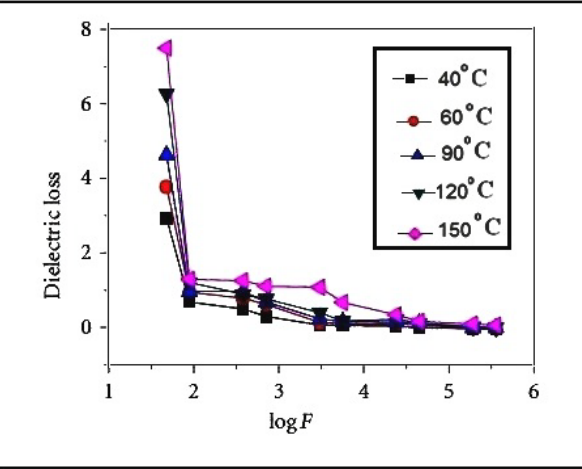

Figure 5: Variation of dielectric loss with frequency.

dielectric loss is strongly dependent on the frequency of the applied field, similar to that of the dielectric constant. The dielectric loss decreases with an increase in the frequency at almost all temperatures. In the low frequency region, high energy loss is observed, which may be due to the dielectric polarization, space charge and rotation direction polarization occurring in the low frequency range [20].

\section{Conclusion}

$\mathrm{MnO}_{2}$ nanoparticles were synthesized by using co-precipitation method. The X-ray diffraction (XRD), scanning electron microscopy (SEM), was used to characterize the structure and morphology of the $\mathrm{MnO}_{2}$ nanoparticles. X-ray diffraction analysis reveals that the crystallite size of the $\mathrm{MnO}_{2}$ nanoparticles was found to be $22 \mathrm{~nm}$. The morphology of the $\mathrm{MnO}_{2}$ nanoparticles was characterized using scanning electron microscopy (SEM). The optical properties were studied by the UV-Visible absorption spectrum. The dielectric constant and dielectric loss of the material decreases with increasing the frequency. In higher frequencies, dielectric constant and dielectric loss are almost constant compared to the lower frequency values.

\section{References}

1. Li WN, Yuan JK, Gomez-Mower S, Xu LP, Sithambaram S, et al. (2006) Hydrothermal synthesis of structure- and shape-controlled manganese oxide octahedral molecular sieve nanomaterials. Adv Funct Mater 16: 1247-1253.

2. Yan JA, Khoo E, Sumboja A, Lee PS (2010) Facile coating of manganese oxide on tin oxide nanowires with high-performance capacitive behavior. ACS Nano. 4: 4247-4255.

3. Deab MS, Ohsaka T (2006) Manganese Oxide Nanoparticles Electrodeposited on Platinum Are Superior to Platinum for Oxygen Reduction. Angew Chem Int Ed 45: 5963-5966.

4. Rault F, Pleyber E, Campagne C, Rochery M, Giraud S, et al. (2009) Effect of manganese nanoparticles on the mechanical, thermal and fire properties of polypropylene multifilament yarn. Polym. Degrad. Stab 94: 955-964.

5. Johan MR, Ting LM (2011) Annealing effects on the properties of copper oxide thin films prepared by chemical deposition. Int. J. Electrochem. Sci., 6: 60946104.

6. Frick R, Muller-Edenborn B, Schlicker A, Rothen-Rutishauser B, Raemy DO et al. (2011) Comparison of Manganese Oxide Nanoparticles and Manganese Sulfate with Regard to Oxidative Stress, Uptake and Apoptosis in Alveolar Epithelial Cells. Toxicol. Lett. 205: 163-172.
7. Wu C, Xie W, Zhang M, Bai L, Yang J, et al. (2009) Environmentally Friendly $\mathrm{Y}-\mathrm{MnO}_{2}$ Hexagon-Based Nanoarchitectures: Structural Understanding and Their Energy-Saving Applications. Chem Eur J 15: 492-500.

8. Cheng FY, Zhao JZ, Song W (2006) Facile controlled synthesis of $\mathrm{MnO} 2$ nanostructures of novel shapes and their application in batteries. Inorganic Chemistry 5: 2038-2044.

9. Xiao W, Wang D, Lou XW (2010) Shape-controlled synthesis of $\mathrm{MnO}_{2}$ nanostructures with enhanced electrocatalytic activity for oxygen reduction. The Journal of Physical Chemistry C 114: 1694-1700.

10. Xu GL, Xu YF, Sun H (2012) Facile synthesis of porous MnO/C nanotubes as a high capacity anode material for lithium ion batteries. Chemical Communications 48: 8502-8504.

11. Yan D, Yan PX, Yue GH (2007) Self-assembled flower-like hierarchical spheres and nanobelts of manganese oxide by hydrothermal method and morphology control of them. Chemical Physics Letters 440: 134-138.

12. Wang X, Li Y (2003) Synthesis and formation mechanism of manganese dioxide nanowires/nanorods. Chemistry A European Journal 9: 300-306

13. Ching S, Welch EJ, Hughes SM, Bahadoor ABF, Suib SL (2002) Nonaqueous sol-gel syntheses of microporous manganese oxides. Chemistry of Materials 14: $1292-1299$.

14. Wang N, Cao X, He L (2008) One-Pot synthesis of highly crystallined $\lambda-\mathrm{MnO}_{2}$ nanodisks assembled from nanoparticles: morphology evolutions and phase transitions. The Journal of Physical Chemistry C 112: 365-369.

15. Oaki Y, Imai H (2007) One-pot synthesis of manganese oxide nanosheets in aqueous solution: chelation-mediated parallel control of reaction and morphology. Angewandte Chemie 46: 4951-4955.

16. Yu X, He Y, Sun J (2009) Nanocrystalline MnO thin film anode for lithium ion batteries with low overpotential. Electrochemical Communications 11: 791-794.

17. Ahmad T, Ramanujachary KV, Lofland SE, Ganguli AK (2004) Nanorods of manganese oxalate: a single source precursor to different manganese oxide nanoparticles ( $\left.\mathrm{MnO}, \mathrm{Mn}_{2} \mathrm{O}_{3}, \mathrm{Mn}_{3} \mathrm{O}_{4}\right)$. Journal of Materials Chemistry 14: 34063410.

18. Harish K, Manisha, Poonam Sangwan (2013) Synthesis and Characterization of $\mathrm{MnO}_{2}$ Nanoparticles using Co-precipitation Technique. International Journal of Chemistry and Chemical Engineering 3: 155-160.

19. Suresh S, Arunseshan C (2014) Dielectric Properties of Cadmium Selenide (CdSe) Nanoparticles synthesized by solvothermal method. Appl Nanosci. 4: 179-184.

20. Sagadevan S (2014) Studies on the dielectric properties of CdS nanoparticles. Appl Nanosci. 4: 325-329. 